\title{
What do we learn from measurements of HOMA-IR?
}

\author{
G. M. Reaven
}

Received: 11 April 2013 /Accepted: 23 April 2013 /Published online: 31 May 2013

(C) Springer-Verlag Berlin Heidelberg 2013

Keywords Glucose $\cdot$ HOMA-IR · Insulin · Insulin resistance

\section{Abbreviation \\ HOMA-IR Homeostasis model assessment of insulin resistance}

To the Editor: Milan Kundera, the great Czech writer, has suggested that all coincidences are meaningful. This view may help explain why the month of April provided us with two excellent research studies, published in the official journals of the EASD [1] and ADA [2], the results of which raise questions as to the biological significance of measurements of the homeostasis model assessment of insulin resistance (HOMA-IR) [3]. HOMA-IR has been widely used as an estimate of insulin sensitivity in cross-sectional, longitudinal and prospective studies, and I believe that the results reported in these manuscripts necessitate reconsideration of what information is provided by this measurement.

The paper by Goldfine et al in Diabetologia [1] examined the effect of salsalate compared with placebo on insulin resistance and glycaemia in impaired fasting glucose and/or impaired glucose tolerance. The results showed that both fasting plasma glucose and insulin concentrations were significantly lower after salsalate treatment. Although the authors did not report HOMA-IR, it is clear from the formula used to calculate this value (fasting glucose $\times$ fasting insulin/constant) that it would have been lower in the salsalate-treated participants. A decrease in HOMA-IR would conventionally be cited as evidence that insulin sensitivity had improved, but direct measurement of insulin-mediated glucose disposal (euglycaemichyperinsulinaemic clamp) showed that there was no change

G. M. Reaven $(\bowtie)$

Falk CVRC, Stanford Medical Center, 300 Pasteur Dive,

Stanford, CA 94395, USA

e-mail: greaven@stanford.edu after salsalate administration. The results of this study also indicated that salsalate decreased insulin clearance, an observation raising additional concerns as to the meaning of HOMA-IR. Specifically, since estimates of HOMA-IR rely on measurement of fasting plasma insulin concentration, any specific concentration will produce the same HOMA-IR, irrespective of whether the insulin concentration was primarily influenced by an increase in pancreatic beta cell secretion or by a decrease in insulin clearance. Thus, the meaning of HOMA-IR can vary according to whether decreased insulin secretion or decreased insulin removal is the major determinant of the change in plasma insulin.

In addressing the somewhat dichotomous finding of lower plasma glucose and insulin concentrations, without any change in insulin sensitivity, the authors raised the possibility that the salsalate-induced changes may have resulted from an effect on basal hepatic glucose production. This suggestion brings into focus the fact that the phrase 'insulin sensitivity (or insulin resistance)' does not have one simple meaning, as well as the complexity of differentiating between 'total body insulin resistance', 'peripheral insulin resistance' and 'hepatic insulin resistance'. These are important issues to be discussed subsequently.

The manuscript in Diabetes Care by Pisprasert and colleagues [2] demonstrates that surrogate estimates of insulin sensitivity can lead to conclusions that are totally different from those based on the results of euglycaemic-hyperinsulinaemic clamp measurements. More specifically, in their studies of non-diabetic individuals they demonstrated that AfricanAmericans were more insulin resistant than Americans of European descent as assessed by a number of surrogate estimates of insulin action. Several such estimates were used, including some based on fasting plasma glucose and insulin concentrations (HOMA-IR [3] and QUICKI [4]), and others using insulin and glucose concentrations resulting from an OGTT (Matsuda index [5] and Stumvoll index [6]). The result of all of these surrogate estimates was that African-Americans are more insulin resistant than European-Americans. However, 
when a euglycaemic-hyperinsulinaemic clamp was performed, no difference in insulin-mediated glucose disposal could be discerned. They also pointed out that in their experimental cohort none of these surrogate estimates of insulin sensitivity was superior to simple measurement of fasting plasma insulin concentrations in predicting insulin sensitivity. Finally, in their discussion of differences between insulin sensitivity measured with the clamp and surrogate estimates, they also addressed the distinction between peripheral as opposed to hepatic insulin resistance.

If HOMA-IR does not measure insulin-mediated glucose disposal as does the clamp, what does it measure? Perhaps the best way to begin is by pointing out that the clamp provides a measure of total body insulin sensitivity: the ability of insulin to stimulate glucose uptake by muscle and inhibit hepatic glucose output. However, as pointed out by Pisprasert and associates [2], in non-diabetic individuals, the degree of hyperinsulinaemia attained during the clamp study is assumed to be high enough to completely inhibit hepatic glucose production. Thus, in this situation, the clamp technique provides a measure of peripheral insulin action.

The fact that HOMA-IR does not provide a very precise estimate of peripheral insulin action seems to have led to the notion that it must be a measure of the ability of insulin to inhibit hepatic glucose production in the fasting state. However, there is no a priori reason to accept this point of view. There are isotopic techniques for measuring hepatic glucose production, and it is possible to conduct experiments to quantify the ability of varying amounts of insulin to inhibit hepatic glucose production. Obviously, such experiments must be performed at a lower insulin concentration than is normally used in euglycaemic-hyperinsulinaemic clamp studies so as not to achieve total inhibition of hepatic glucose production in every participant. To the best of my knowledge, no such studies have been performed to define the degree of correlation between calculation of HOMA-IR and direct measurement of insulin inhibition of hepatic glucose production. Until that has been accomplished, it seems to me that considerable caution should be exercised in using HOMA-IR as an accurate measure of total body, peripheral or hepatic insulin resistance.

Duality of interest The author declares that there is no duality of interest associated with this manuscript.

Contribution statement The author was the sole contributor to this paper.

\section{References}

1. Goldfine AB, Conlin PR, Halperin F et al (2013) A randomised trial of salsalate for insulin resistance and cardiovascular risk factors in persons with abnormal glucose tolerance. Diabetologia 56:714-723

2. Pisprasert V, Ingram KH, Lopez-Davila MF, Munoz AJ, Garvey WT (2013) Limitations in the use of indices using glucose and insulin levels to predict insulin sensitivity: impact of race and gender and superiority of the indices derived from oral glucose tolerance test in African Americans. Diabetes Care 36:845-853

3. Matthews DR, Hosker JP, Rudenski AS, Naylor BA, Treacher DF, Turner RC (1985) Homeostasis model assessment: insulin resistance and beta-cell function from fasting plasma glucose and insulin concentrations in man. Diabetologia 28:412-419

4. Katz A, Nambi SS, Mather K et al (2000) Quantitative Insulin Sensitivity Check Index: a simple, accurate method for assessing insulin sensitivity in humans. J Clin Endocrinol Metab 85:24022410

5. Matsuda M, DeFronzo RA (1999) Insulin sensitivity indices obtained from oral glucose tolerance testing: comparison with the euglycemic insulin clamp. Diabetes Care 22:1462-1470

6. Stumvoll M, Mitrakou A, Pimenta W et al (2000) Use of the oral glucose tolerance test to assess insulin release and insulin sensitivity. Diabetes Care 23:295-301 